ARTICLE

\title{
Structural reorganization of cylindrical nanoparticles triggered by polylactide stereocomplexation
}

Liang Sun ${ }^{1}$, Anaïs Pitto-Barry ${ }^{1}$, Nigel Kirby ${ }^{2}$, Tara L. Schiller ${ }^{3,4}$, Ana M. Sanchez ${ }^{5}$, M. Adam Dyson ${ }^{5}$, Jeremy Sloan ${ }^{5}$, Neil R. Wilson ${ }^{5}$, Rachel K. O'Reilly ${ }^{1} \&$ Andrew P. Dove ${ }^{1,3}$

Co-crystallization of polymers with different configurations/tacticities provides access to materials with enhanced performance. The stereocomplexation of isotactic poly(L-lactide) and poly( $D$-lactide) has led to improved properties compared with each homochiral material. Herein, we report the preparation of stereocomplex micelles from a mixture of poly(L-lactide)-b-poly(acrylic acid) and poly(D-lactide)-b-poly(acrylic acid) diblock copolymers in water via crystallization-driven self-assembly. During the formation of these stereocomplex micelles, an unexpected morphological transition results in the formation of dense crystalline spherical micelles rather than cylinders. Furthermore, mixture of cylinders with opposite homochirality in either THF/ $\mathrm{H}_{2} \mathrm{O}$ mixtures or in pure water at $65^{\circ} \mathrm{C}$ leads to disassembly into stereocomplexed spherical micelles. Similarly, a transition is also observed in a related PEO- $b$-PLLA/PEO-b-PDLA system, demonstrating wider applicability. This new mechanism for morphological reorganization, through competitive crystallization and stereocomplexation and without the requirement for an external stimulus, allows for new opportunities in controlled release and delivery applications.

\footnotetext{
${ }^{1}$ Department of Chemistry, University of Warwick, Coventry CV4 7AL, UK. ${ }^{2}$ Australian Synchrotron, 800 Blackburn Road, Clayton, Victoria 3168, Australia. ${ }^{3}$ Department of Materials Engineering, Monash University, Clayton, Melbourne, Victoria 3800, Australia. ${ }^{4}$ School of Science and Technology, University of New England, New England, New South Wales 2351 Australia. ${ }^{5}$ Department of Physics, University of Warwick, Coventry CV4 7AL, UK. Correspondence and requests for materials should be addressed to R.K.O'R. (email: r.k.o-reilly@warwick.ac.uk) or to A.P.D. (email: a.p.dove@warwick.ac.uk).
} 
P olylactide has aroused great interest in the biomedical and pharmaceutical fields in the past few decades owing to its outstanding biodegradability, biocompatibility and low toxicity ${ }^{1-5}$. The cyclic lactide monomer presents two stereocenters and thus provides three stereoisomeric forms: $L$-lactide, $D$-lactide and meso-lactide. The polymerization of $L$-lactide or $D$-lactide leads to the formation of isotatic poly(L-lactide) (PLLA) and poly(D-lactide) (PDLA), which can co-crystallize to form the stereocomplex between the two polymer chains with opposite configurations. The stereocomplexation of polylactide was reported in 1987 by Ikada et al. ${ }^{6}$ who used differential scanning calorimetry and wide-angle X-ray diffraction (WAXD) to study the stereocomplexation of different ratios of PLLA and PDLA blends. It has been reported that the stereocomplex polylactide crystallites are stabilized by the $\mathrm{CH}_{3} \cdots \mathrm{O}=\mathrm{C}$ hydrogen-bonding interaction between the two opposite homochiral polymer chains, allowing a more compact conformation and a more dense polymeric packing than homochiral crystallites ${ }^{7}$. Stereocomplexation is of great interest in materials science, as the stereocomplex formed between the left-handed PLLA and right-handed PDLA polymeric helices has been shown to possess improved mechanical and thermal properties when compared with the homochiral polymers ${ }^{2,6,8,9}$. Beyond the stereocomplex formation in homopolymer blends, the self-assembly of polylactide (PLA)-containing block copolymers in selective solvents has opened up substantial opportunities to fabricate various nanoparticles with optimized properties ${ }^{10-17}$. For instance, stereocomplexation of poly( $($-lactide) $-b$ poly(ethylene glycol) (PLLA- $b$-PEG) and poly(D-lactide)- $b$ poly(ethylene glycol) (PDLA- $b$-PEG) diblock copolymers resulted in enhanced stability of the resultant micellar constructs $^{12}$. Furthermore, the stereocomplex micelles prepared from Y-shaped PEG- $b$-PLLA- $b$-PLLA and PEG- $b$-PDLA- $b$ PDLA miktoarm copolymers showed high loadings of paclitaxel and enhanced stability ${ }^{4}$. The stereocomplexation of PLLA and PDLA has also enabled access to a wide variety of nanoparticle morphologies ${ }^{15,16}$. Another approach, in which the stability has been predicted by theoretical simulations ${ }^{18,19}$, uses a mixed micelle approach where block copolymers with complementary blocks that have selective interactions between either the core or shell-forming blocks enable the access to a range of morphologies ${ }^{20,21}$. This concept has also been applied to PLAcontaining materials, for example, Hedrick and co-workers obtained patchy polymeric micelles from the mixture of poly $(L-$ lactide)- $b$-poly( $N$-isopropylacrylamide) (PLLA- $b$-PNIPAAM) and PDLA- $b$-PEG diblock copolymers ${ }^{13}$, whereas Bouteiller and researchers observed cylindrical micelles by mixing poly $\left(L^{-}\right.$ lactide)- $b$-poly( $\varepsilon$-caprolactone) and poly(D-lactide)- $b$-poly( $\varepsilon$ caprolactone) in tetrahydrofuran $(\mathrm{THF})^{10,17}$. In our previous studies $^{22-25}$, we have shown PLLA- $b$-poly(acrylic acid) or PDLA$b$-PAA diblock copolymers with a large hydrophilic weight fraction $(82 \%)$ can assemble into homochiral cylindrical micelles through a crystallization-driven self-assembly (CDSA) process in solution above the glass transition temperature of the PLA block (typically $\left.55-60^{\circ} \mathrm{C}\right)^{22}$. These cylindrical micelles were also able to load hydrophobic dyes inside their hollow cores, which were induced by the hydrogen-bonding interaction between PLLA core and PAA corona during the slow-drying process ${ }^{23}$.

Herein, we report our studies into the application of stereocomplexation in CDSA processes. In an attempt to form more stable cylinders through stereocomplexation, we explore the assembly of a mixture of PLLA- $b$-PAA and PDLA- $b$-PAA diblock copolymers, under the same assembly conditions and surprisingly observed the formation of spherical micelles with stereocomplex cores. Interestingly, when mixed under CDSA conditions, the two homochiral cylinders undergo a morphological transition into spherical micelles with a PLA stereocomplex core. This transformation, in which cylinders transform into spheres, is the opposite of what is usually observed and as such is a rare observation; this is more so given that the core of the micelles in this system is highly crystalline ${ }^{26}$. This stereocomplex-induced reorganization is also demonstrated for a biocompatible PEG- $b$-PLA block copolymer system, as well as through the addition of a unimer solution to a solution of cylinders of opposite homochirality. This new mechanism of structural reorganization provides a potential new trigger for controlled/ targeted drug release in polymeric vehicles.

\section{Results}

Synthesis of the precursor diblock copolymers. Diblock copolymers used in this study were achieved by a combination of ring-opening polymerization (ROP) of either $L$ - or $D$-lactide with a highly selective metal-free thiourea/(-)-sparteine co-catalyst system and reversible addition-fragmentation chain transfer polymerization of tetrahydropyran acrylate (THPA) monomer from a dual-headed initiator as reported previously ${ }^{22,25}$. The compositions of the precursor diblock copolymers were determined by ${ }^{1} \mathrm{H}$ NMR spectroscopy, resulting in PLLA ${ }_{31}-b$ PTHPA $_{332}$ (1) and PDLA $_{28}-b-$ PTHPA $_{315}$ (2). All the polymers used in this study possess low dispersities $\left(\oplus_{\mathrm{M}}<1.20\right)$, determined by size exclusion chromatography (SEC) analysis. Their characterization details are available in the Supplementary Information (Supplementary Figs $1-7$ and Supplementary Table 1).

Self-assembly of diblock copolymers. The exploration of the stereocomplex formation of PLLA- $b$-PAA and PDLA- $b$-PAA was conducted using the CDSA conditions reported previously ${ }^{22,25}$ but with a mixture of the two homochiral diblock copolymers. Specifically, 1 and 2 were mixed in an equal mass ratio in a vial before addition of a mixture of THF and $\mathrm{H}_{2} \mathrm{O}\left(v_{\mathrm{THF}} / v_{\mathrm{H} 2 \mathrm{O}}=\right.$ 20/80), giving the final concentration of diblock copolymers of $20 \mathrm{mg} \mathrm{ml}^{-1}$. Acetic acid was added into the mixture to promote the hydrolysis of PTHPA into PAA and hence allow the formation of amphiphilic diblock copolymers. The sealed vial was pierced with a needle on top to allow the evaporation of THF during the self-assembly process. The temperature of the selfassembly was $65^{\circ} \mathrm{C}$, which is above the $T_{\mathrm{g}}$ of polylactide ${ }^{27}$, to allow the PLA blocks to move more freely and thus promote the co-crystallization of PLLA and PDLA. We have previously demonstrated that the PTHPA block can be completely converted into PAA within the first $2 \mathrm{~h}$ of the self-assembly ${ }^{24}$.

After $30 \mathrm{~h}$ of self-assembly, spherical micelles $\left(D_{\mathrm{n}}=50 \pm 7 \mathrm{~nm}\right)$ were observed using transmission electron microscopy (TEM) (Fig. 1c). The observed spherical morphology was not expected as under the same self-assembly conditions, the homochiral PLLA$b$-PAA (or PDLA-b-PAA) diblock copolymer leads to the formation of a cylindrical morphology (Fig. 1a,b) ${ }^{22-25}$. We had hypothesized that a more stable cylindrical structure would form as a result of the stereocomplexation of the diblock copolymers bearing PLLA or PDLA blocks as observed in a related system by Boutellier and co-workers ${ }^{10,17}$. WAXD was used to confirm the successful co-crystallization of the two enantiomeric PLA blocks in the core domain. An intense Bragg peak at a $2 \theta$ value of $12^{\circ}$ (which belongs to the (001) plane) definitively proved the crystalline stereocomplex formation (Fig. 1f), whereas the two homochiral cylinders obtained from 1 and 2, respectively, showed a completely different crystalline Bragg peak at a $2 \theta$ value of $16.6^{\circ}$ (which belongs to the (110)/(200) planes) (Fig. 1d,e e)

The self-assembly process for the formation of stereocomplex spheres was further investigated by fourier transfom-infrared 

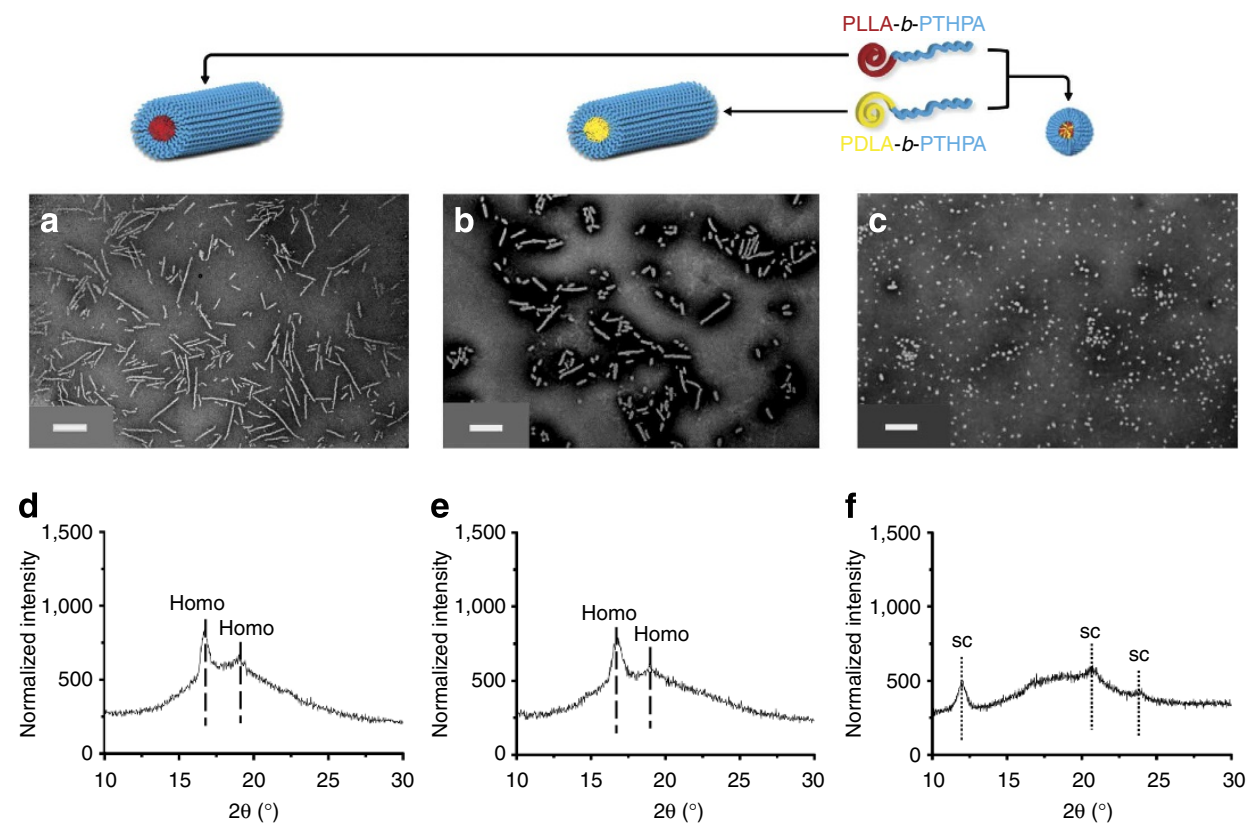

Figure 1 | Characterization data of the nanostructures obtained from the self-assembly of diblock copolymers $\mathbf{1}$ or/and $\mathbf{2}$. Assembly performed in $\mathrm{THF} / \mathrm{H}_{2} \mathrm{O}(20 / 80 \mathrm{v} / \mathrm{v})$ at $65^{\circ} \mathrm{C}$. (a) TEM image of cylindrical micelles achieved from the self-assembly of the L-enantiomer 1 ; (b) TEM image of cylindrical micelles achieved from the self-assembly of the $D$-enantiomer 2; (c) TEM image of spherical micelles obtained from the mixture of the $L$ and $D$-enantiomers 1 and $2 ;(\mathbf{d}, \mathbf{e})$ The homochiral cylinders showing Bragg peaks at $2 \theta$ values of $16.6^{\circ}$ and $19.2^{\circ}$ from WAXD diffractograms; (f) Stereocomplex spheres gave Bragg peaks at $2 \theta$ values of $12^{\circ}, 20.6^{\circ}, 23.8^{\circ}$. All assemblies were run for $30 \mathrm{~h}$ before analysis. TEM samples were prepared by slow drying and negatively stained using phosphotungstic acid (PTA). Scale bars $=500 \mathrm{~nm}$.

a

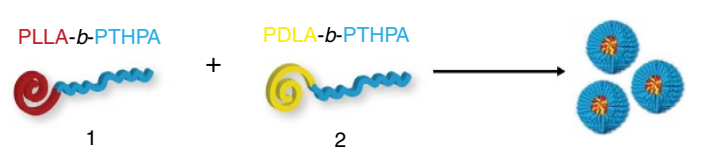

C
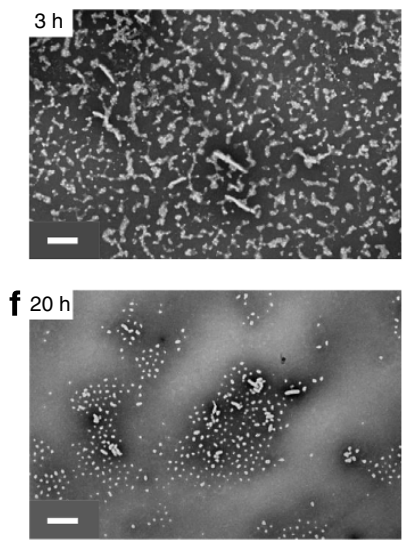

d
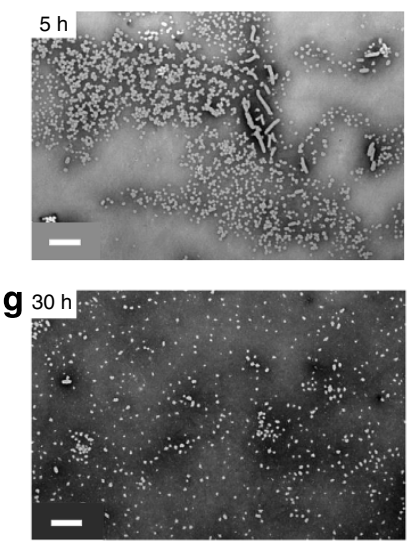

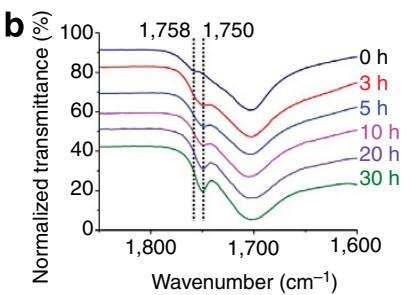

e
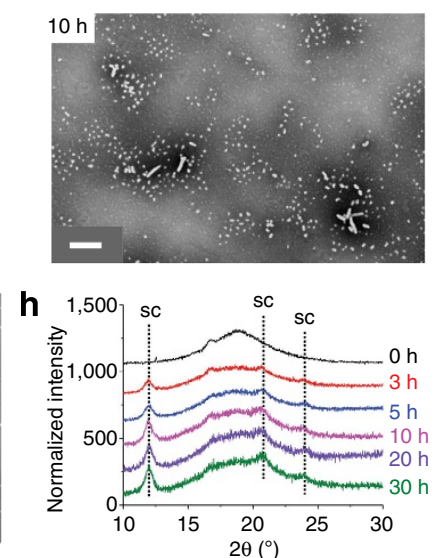

Figure 2 | Characterization of the formation of the stereocomplex spherical micelles from the homochiral polymers $\mathbf{1}$ and $\mathbf{2}$. Studies conducted in $\mathrm{THF} / \mathrm{H}_{2} \mathrm{O}(2 \mathrm{O} / 80 \mathrm{v} / \mathrm{v})$ at $65^{\circ} \mathrm{C}$ as a function of time. (a) Cartoon illustration of the formation of stereocomplex spherical micelles from 1 and 2. (b) FT-IR spectra of dried nanoparticles revealed that the wavenumber of carbonyl vibrational stretch of polylactide shifted from 1,758 to $1,750 \mathrm{~cm}^{-1}$ over time. TEM images: (c) $3 \mathrm{~h}$ after starting the self-assembly; (d) after $5 \mathrm{~h}$; (e) after $10 \mathrm{~h}$; (f) after $20 \mathrm{~h}$ and (g) after $30 \mathrm{~h}$. (h) WAXD diffractograms showed that the Bragg peak for stereocomplex formation between 1 and 2 increased over time. Cylindrical micelles obtained from the homochiral diblock copolymers 1 or 2 were found to decrease in length during the self-assembly $(\mathbf{c}-\mathbf{g})$. TEM samples were prepared by slow drying and negatively stained using PTA. Scale bars $=500 \mathrm{~nm}$. 
a

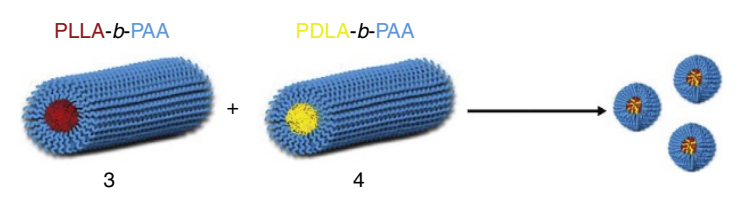

C

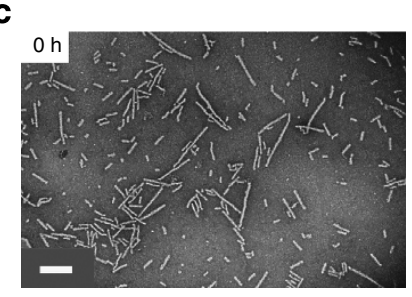

d
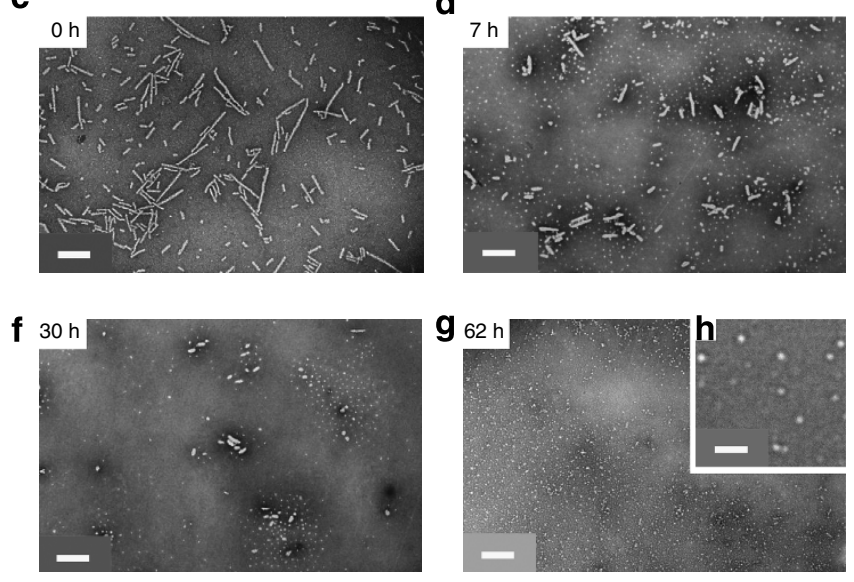

b

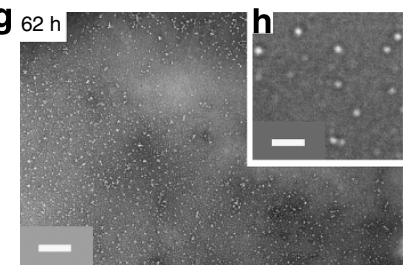

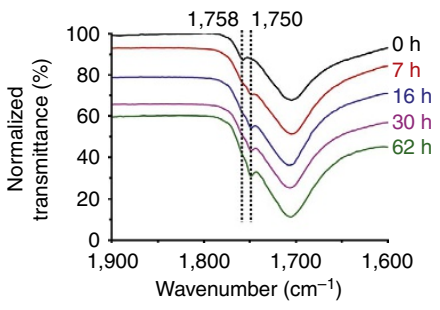

e
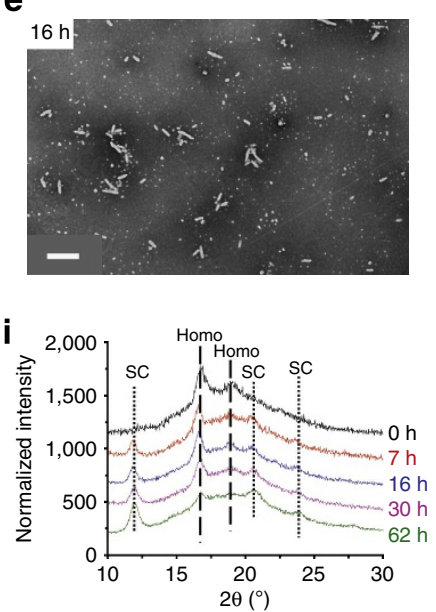

Figure 3 | Morphological transition and the changes to the crystallinity on mixing of two homochiral cylinders 3 and 4 . Conducted at $65^{\circ} \mathrm{C}$ with the addition of fresh THF (final solvent composition THF/ $\mathrm{H}_{2} \mathrm{O} 20 / 80 \mathrm{v} / \mathrm{v}$ ). (a) Cartoon illustration showing the morphological transition from homochiral cylinders 3 and 4 to stereocomplex spheres. Scale bar $=500 \mathrm{~nm}$ (b) FT-IR spectra of dried nanoparticles, which reveal the wavenumber of carbonyl group vibration of poly(lactide) shifted from 1,758 to $1,750 \mathrm{~cm}^{-1}$ over time. Scale bar $=500 \mathrm{~nm}$. (c-g) TEM images, which illustrate the length of the cylindrical micelles decreased while the population of spherical micelles increased over time. Scale bars $=500 \mathrm{~nm}$. (h) TEM expansion of $62 \mathrm{~h}$ time point. Scale bar $=100 \mathrm{~nm}$. (i) WAXD diffractograms illustrating that the intensity of stereocomplex Bragg peak at a $2 \theta$ value of $12^{\circ}$ increased gradually, whereas the intensity of homochiral Bragg peak at a $2 \theta$ value of $16.6^{\circ}$ decreased significantly over time. TEM samples were prepared by slow drying and negatively stained using PTA.

(FT-IR) spectroscopy using dried nanoparticle samples (Fig. 2b). The vibrational stretch of the carbonyl group of polylactide was found to shift to a lower wavenumber (from 1,758 to $1,750 \mathrm{~cm}^{-1}$ ) after $3 \mathrm{~h}$ (Fig. 2b). Such a shift is attributed to the arrangement of the polylactide chains from a disordered state to an ordered one throughout the stereocomplex formation ${ }^{10,17}$. By using WAXD (Fig. 2h), an obvious stereocomplex Bragg peak at a $2 \theta$ value of $12^{\circ}$ is observed after $3 \mathrm{~h}$, which indicates a fast formation of the stereocomplex micelles. Prolonged assembly under these conditions promoted significantly the stereocomplexation of 1 and 2 as well as the slight increase in diameter of these spheres (from $42 \pm 7 \mathrm{~nm}$ in Fig. $2 \mathrm{~d}$ to $50 \pm 7 \mathrm{~nm}$ in Fig. 2g, respectively). A careful study of the TEM images during the self-assembly process (Fig. 2c-g) revealed an interesting phenomenon: a number of cylinders existed at the beginning of the self-assembly, but their lengths gradually decreased, and as such, the cylindrical morphology almost disappeared after $30 \mathrm{~h}$. As the cylinders are believed to only self-assemble from the homochiral diblock copolymer 1 or 2 but not from the stereocomplex, we propose that the crystallization process is fast and leads to the resolution of the mixture to $D$ and $L$ homochiral cylinders, which over time undergo a morphological transition into stereocomplex spheres. Such a morphological transition is unusual and only one example has been previously reported $^{26}$. Indeed, this observation is in contrast to that observed by Boutellier and coworkers ${ }^{10,17}$ in which mixtures of poly( $\varepsilon$-caprolactone)- $b$-PLLA and poly( $\varepsilon$-caprolactone)- $b$-PDLA copolymers were self-assembled into cylinders in THF. These differences in behaviour are attributed to an absence of selective solvation and more similar block volume ratios than applied in this work.

Stereocomplexation triggered morphological transitions. To explore the morphological transition in more detail and demonstrate its potential utility, separate solutions of homochiral cylinders $3\left(L_{\mathrm{n}}=194 \mathrm{~nm}, L_{\mathrm{w}} / L_{\mathrm{n}}=1.22, W_{\mathrm{n}}=53 \pm 6 \mathrm{~nm}\right)$ and 4 $\left(L_{\mathrm{n}}=188 \mathrm{~nm}, L_{\mathrm{w}} / L_{\mathrm{n}}=1.21, W_{\mathrm{n}}=54 \pm 7 \mathrm{~nm}\right)$ were prepared from diblock copolymers 1 and 2, respectively, (Fig. 1a,b) under CDSA conditions for $30 \mathrm{~h}$ as described above. Subsequently, $1 \mathrm{ml}$ (around $17 \mathrm{mg} \mathrm{ml}^{-1}$ ) of each cylinder solution (in pure water) was mixed together with an additional $0.5 \mathrm{ml}$ of THF to the cylinder mixture to mimic the starting self-assembly conditions described above $\left(v_{\mathrm{THF}} / v_{\mathrm{H} 2 \mathrm{O}}=20 / 80\right)$. The solution was then heated at $65^{\circ} \mathrm{C}$ with a needle added through the cap to allow the THF to evaporate (Fig. 3a). By using such an approach, we observed a marked decrease in cylinder lengths and increase in the population of spherical micelles by TEM (Fig. 3c-g), which demonstrates the ability to trigger the morphological transition through the onset of stereocomplexation. Analysis by dynamic light scattering (DLS) also illustrated the decrease in the hydrodynamic diameters of the nanoparticles over time (Supplementary Fig. 8). In situ synchrotron small-angle X-ray scattering (SAXS) further confirmed the cylinder-to-sphere transition (Fig. 4). A comparison of the parameters obtained from the fitted SAXS curves (Supplementary Table 2 and Supplementary Fig. 9) revealed the presence of an increasing number of spheres along with a decreasing dimension of 
a

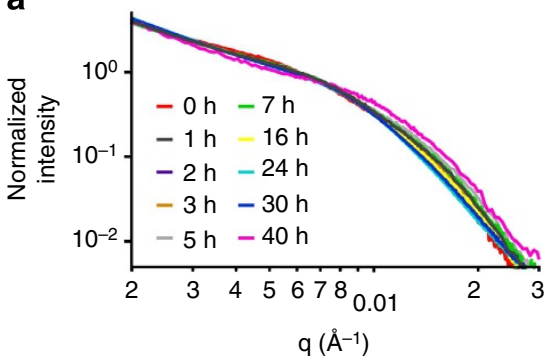

C

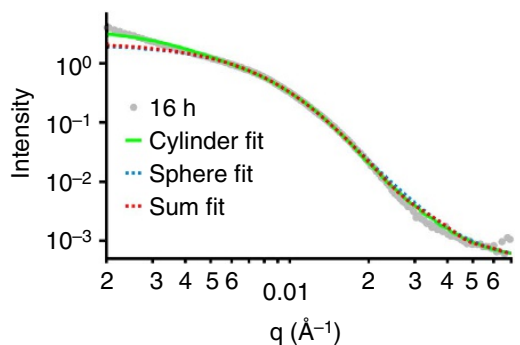

b

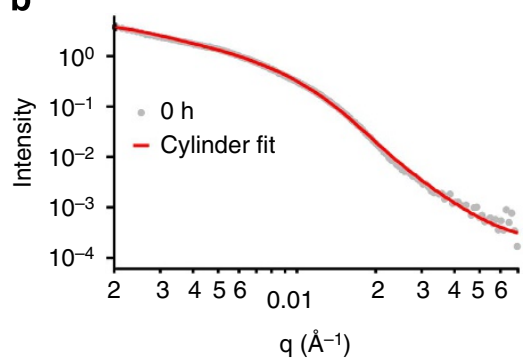

d

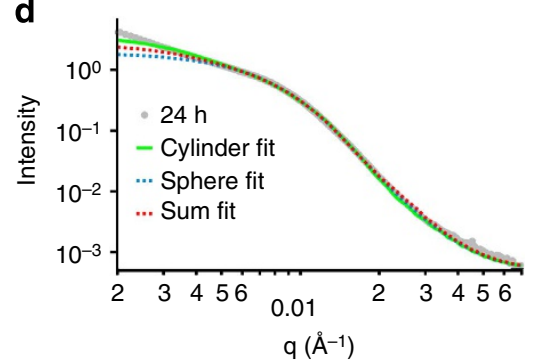

Figure 4 | SAXS experimental profiles and fittings of the morphological transition from the two homochiral cylinders 3 and 4 to stereocomplex spheres. Conducted at $65^{\circ} \mathrm{C}$ with the addition of fresh THF at the beginning of the self-assembly. (a) SAXS experimental profiles during the entire self-assembly process: a change of morphology is observed, with a decrease of the radius of the morphology as the curve inflexions move to higher $q$-values with time. (b-d) Fitting of some experimental profiles with different models: cylinder (b) and a linear combination of sphere and cylinder (c,d), see also Supplementary Table 2.

cylinders over time. These observations were in accordance with TEM and DLS results.

FT-IR spectroscopic analysis was performed on samples taken throughout the cylinder-to-sphere transition to prove that the stereocomplex formation had occurred (Fig. 3b). A lower wavenumber shift from 1,758 to $1,750 \mathrm{~cm}^{-1}$ was observed for the vibration of the carbonyl group of the polylactide chains, which suggests the successful formation of stereocomplex micelles. By using WAXD (Fig. 3i), a significant decrease in the intensity of the homochiral peak at a $2 \theta$ value of $16.6^{\circ}$ and a gradual intensity increase of the stereocomplex peak at $2 \theta$ value of $12^{\circ}$ were observed. Importantly, the same morphological transition from homochiral cylinders to stereocomplex spheres was also observed when 3 and 4 were mixed and heated without the addition of fresh THF as evidenced by TEM, albeit over a longer time period (Supplementary Figs 10c-10g), DLS (Supplementary Fig. 11), SAXS (Supplementary Fig. 12 and Supplementary Table 3), FT-IR (Supplementary Fig. 10b) and WAXD (Supplementary Fig. 10h). Furthermore, following complete removal of THF from the system by freeze-drying the homochiral cylinders, the morpohological transition was still observed by the expected change in particle morphology by TEM and DLS (Supplementary Fig. 13). We propose that the stereocomplexation is hindered without the presence of a good solvent for polylactide (such as THF), which assists in chain folding and the co-crystallization in the core domain. The dimensions of the stereocomplex spheres synthesized from unimers $\left(D_{\mathrm{n}}=49.7 \mathrm{~nm}\right.$, Fig. 1c) are much larger (more than twice) than those of stereocomplex spheres achieved from disassembly of cylinders $\left(D_{\mathrm{n}}=22 \pm 3 \mathrm{~nm}\right.$ for Fig. $3 \mathrm{~g}, D_{\mathrm{n}}=20 \pm 5$ $\mathrm{nm}$ for Supplementary Fig. 10g), as indicated by TEM analysis. As the stereocomplex core and PAA corona are both visible after staining with phosphotungstic acid (PTA), the crystalline stereocomplex core domain appears even smaller than that observed using negative staining.

To further compare the stereocomplex spherical micelle (Fig. 5a, the same sample shown in Fig. 3g) and amorphous spherical micelle (Fig. 5e, obtained from the self-assembly of 1 , the preparation method is given in Methods, WAXD in Supplementary Fig 14), we used an aberration-corrected high resolution TEM at near focus condition using graphene oxide (GO) supports, which are nearly electron transparent ${ }^{28}$. Exit wave reconstruction (EWR) enabled a higher contrast and higher resolution image to be obtained from a focal series of TEM images, despite the low contrast of predominantly carbon-based materials, and thus provided more structural detail for the macromolecular nanostructures ${ }^{29}$. EWR gives a phase image and an amplitude image; macromolecular nanostructures exhibit mainly phase contrast in the TEM and so are clearly resolved in the phase images. The square of the amplitude image (the intensity) gives contrast, which is roughly related to the mass thickness of the macromolecular sample. By using EWR on unstained samples, a much clearer spherical shape was observed for both stereocomplex and amorphous micelles in the phase (Supplementary Fig. 15). The squared amplitude images from EWR (Fig. 5b,f) revealed that the stereocomplex spherical micelle possessed a higher density than the amorphous spherical micelle, with the intensity relative to the background decreased by $8 \%$ for the former but only $3 \%$ for the latter (Fig. 5c,g) (decreased intensity is due to increased scattering of the electron beam and hence implies increased mass thickness). The surface plots of EWR squared amplitude images (Fig. 5d,h) demonstrate the higher density of the stereocomplex micelle and thus provide further evidence of its altered physical state.

To prove that the effects observed were a consequence of the onset of stereocomplexation, two control experiments were conducted using identical self-assembly conditions as described above, to monitor the changes in the homochiral cylinder 3 only. The single enantiomer, homochiral cylindrical micelles were heated at $65^{\circ} \mathrm{C}$ for an extended time at a high concentration $\left(17 \mathrm{mg} \mathrm{ml}^{-1}\right)$. With no addition of fresh THF, the length of these cylinders stayed approximately the same even after $120 \mathrm{~h}$ of heating, although some aggregation was observed by TEM analysis (Supplementary Figs 16a-16c). This observation 

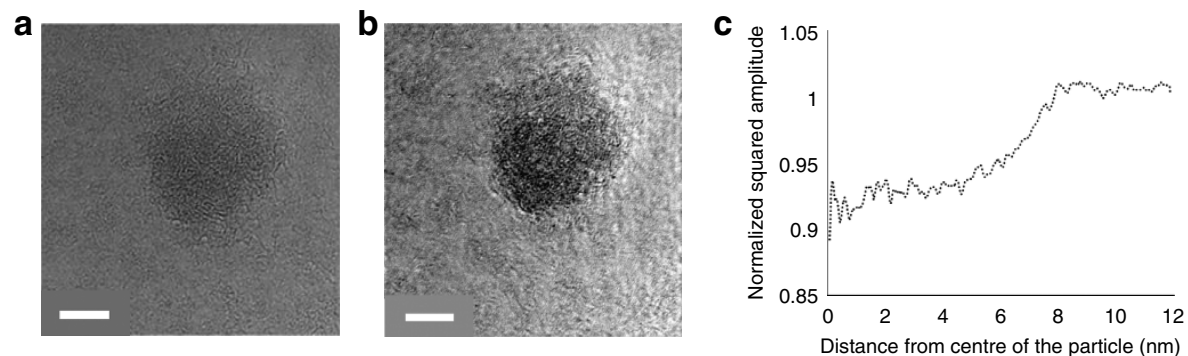

d
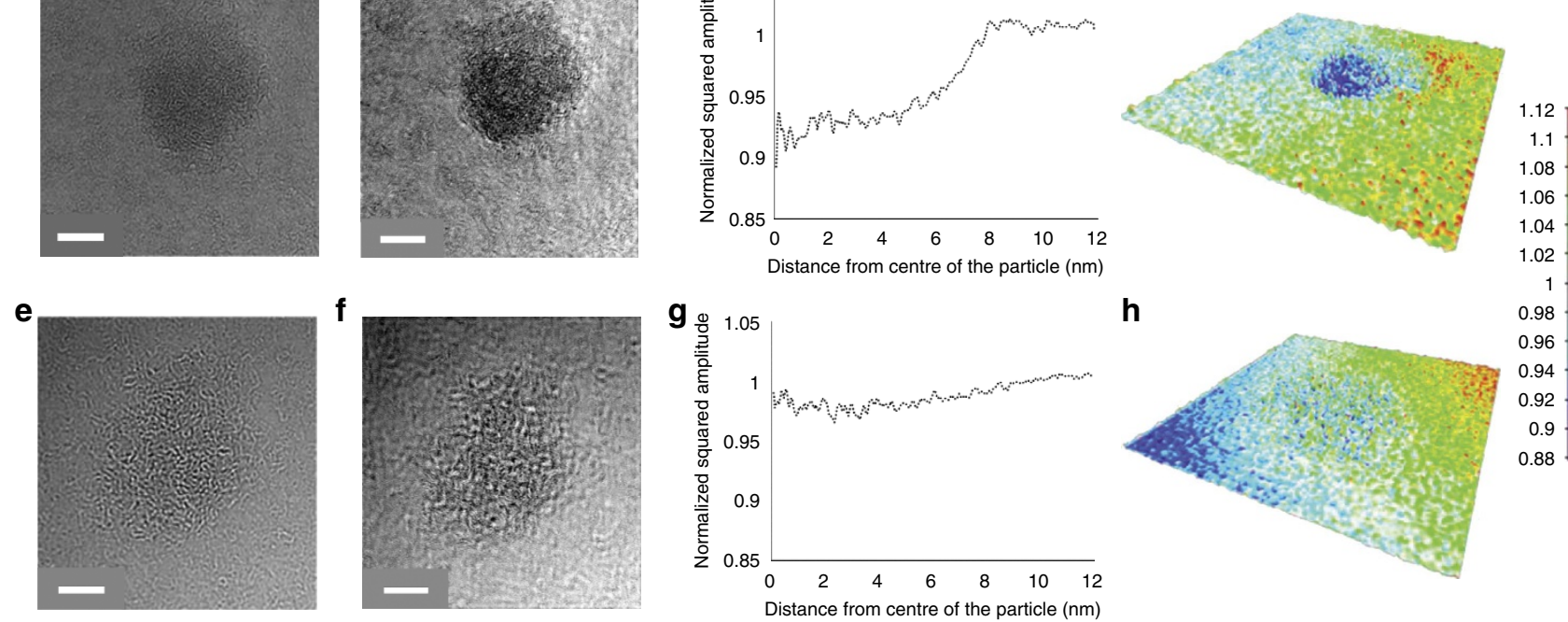

h
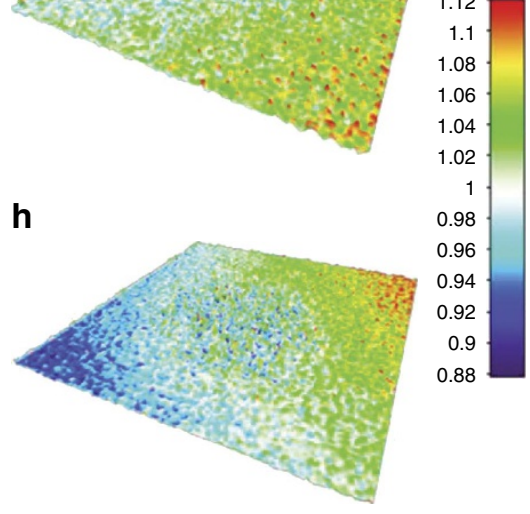

Figure 5 | Microscopy analysis of stereocomplex and amporphous spherical micelles. (a-d) A stereocomplex spherical micelle (obtained from the mixture of homochiral cylinders 3 and 4 ) and (e-h) an amorphous spherical micelle (obtained from the self-assembly of 1). (a) Near focus aberrationcorrected microscope image and (b) EWR-squared amplitude image of a stereocomplex sphere; (e) Near focus aberration-corrected microscope image and (f) EWR-squared amplitude image of an amorphous sphere. All of the images were obtained on graphene oxide supports. The increased density of the stereocomplex sphere relative to the amorphous sphere can be seen in the stronger contrast in the squared amplitude image, this is shown quantitatively in panels $\mathbf{c}$ and $\mathbf{g}$ (for the stereocomplex micelle and amorphous micelle, respectively), which plots the radially averaged profile of the squared amplitude from the middle of each sphere to the GO support. Panels $\mathbf{d}$ and $\mathbf{h}$ show the surface plots of the EWR-squared amplitude images demonstrate the higher density of the stereocomplex micelle. Scale bars $=5 \mathrm{~nm}$.

was supported by SAXS analysis (Supplementary Fig. 17, Supplementary Table 4), which also indicated unchanged cylinder length as well as some aggregation after excessive heating. Moreover, no obvious changes in the intensity of the Bragg peak at a $2 \theta$ value of $16.6^{\circ}$ were observed from WAXD diffractograms over time (Supplementary Fig. 16d), again indicating the high stability of these cylinders. Interestingly, when the cylinder solution was exposed to fresh THF $\left(v_{\mathrm{THF}} / v_{\mathrm{H} 2 \mathrm{O}}=20 / 80\right)$ at $65^{\circ} \mathrm{C}$, with a needle through the seal of the vial, the cylinder length decreased rapidly within the first few hours but then large lamellar particles were formed after $16 \mathrm{~h}$, as observed by TEM analysis (Supplementary Figs 18a-18e). The formation of platelets rather than cylinders on addition of a common solvent has been detected previously for crystalline-coil systems ${ }^{30}$. These trends were further confirmed by SAXS with an observed increase in the population of lamellar particles compared with the population of cylinders over the heating process (Supplementary Fig. 19 and Supplementary Table 5). WAXD diffractograms of this system also indicated that the intensity of the homochiral peak at the $2 \theta$ value of $16.6^{\circ}$ decreased after the addition of fresh THF into the cylinder solution but recovered after another $30 \mathrm{~h}$ of heating under the self-assembly conditions (Supplementary Fig. 18f). The lack of cylinder-to-sphere transition was confirmed by TEM and SAXS during these two control experiments.

The stereocomplexation-triggered morphological transition was also investigated for a fully biocompatible PLA system (PLLA- $b$-PEG and PDLA- $b$-PEG (Supplementary Figs 20 and $21)$ ), which was synthesized as we have reported previously ${ }^{31}$. Over a similar timeframe, the PLA-b-PEG copolymer system also demonstrated a transition from crystalline cylinders to stereocomplex spheres. We suggest that this new mechanism for nanostructure reorganization, which is triggered by stereocomplexation, highlights the potential utility of these PLA materials in controlled release or delivery applications across a wide range of applications.
Explanation of the morphological transition. The exact mechanism of cylinder growth in this and related systems is not well understood, with two possible mechanisms suggested by Eisenberg et al. ${ }^{32}$ in the late 1990s: the unimer-exchange theory, in which cylindrical nanoparticles are proposed to grow by an exchange of unimers between particles, and the adhesive fusion theory, in which it is proposed that micelles collide together and adhere to one another to form cylinders. Although much evidence to date has supported the adhesive fusion mechanism $^{33,34}$, several in-depth studies from the Manners, Winnik and Schmalz groups have suggested that a unimerexchange mechanism occurs to some extent when epitaxial growth of crystalline-core cylinders occurs at elevated temperature (in a selective solvent) or at room temperature (on addition of a common solvent to the selective solvent) ${ }^{35-39}$. In the present study, the morphological transition from homochiral cylinders into stereocomplex spheres is somewhat unexpected given that under thermodynamic control, mixed micelle formation between two polymers of such structural similarity would be expected to retain the cylindrical morphology. The stereocomplexation driving force that exists in this system changes the crystallization behaviour of the core-forming blocks and hence is able to drive this morphology switch. Importantly, these results are highly consistent with the existence of unimers in solution under assembly/disassembly conditions. When the cylinder solutions were mixed, stereocomplex micelles formed gradually from these dynamic unimers with both the $L$ and $D$ unimers locked into the stereocomplex micelles. Only by being present as unimers in solution could the components of different stereochemistry interact sufficiently to form stereocomplex micelles that display a higher stability owing to the improved packing and strong interactions of the helical chains of opposite configurations $s^{2,6,7,40}$. In an adhesive fusion process, homochiral cylinders would be expected to form when 3 and 4 were mixed, however, this phenomenon was not observed. Such a unimer- 
exchange process was further confirmed when the addition of $D$-unimers (dissolved in THF) into aqueous solution of $L$-cylinders (3) led to the disassembly of the latter and the formation of the stereocomplex spherical micelles (Supplementary Fig. 22). Although the disassembly is accelerated by the addition of a common solvent for both blocks, its observation provides a potential new trigger to induce a morphological transition or release from nanoscale particles in a completely aqueous system.

In summary, we have demonstrated that stereocomplexation can be used to trigger the reorganization of homochiral PLAcontaining cylindrical micelles to form small stereocomplex spherical micelles, in contrast to that usually observed in CDSA systems. This morphology transition has been monitored by TEM, DLS, in situ synchrotron SAXS, WAXD and FT-IR analysis. Such a morphological transition was not observed when enantiopure PLLA- $b$-PAA cylinders were exposed to the same self-assembly conditions without the presence of the opposite enantiopure PDLA- $b$-PAA cylinders. Both the mechanism of the cylinder-to-sphere transition in this system and the homochiral cylinder growth were confirmed to occur by a unimer-exchange process. We propose that this fundamental study provides a deeper understanding of the balance of self-assembly forces in such PLA systems and suggest that this stereocomplexationtriggered reorganization may have potential applications in delivery/controlled release.

\section{Methods}

Materials. Chemicals and solvents were used as purchased from Aldrich, Acros, Fluka, Fisher Chemical, Alfa Aesar or VWR. $L$-Lactide and $D$-lactide monomers were kindly donated by Corbion-Purac and were passed through a silica plug with dichloromethane $\left(\mathrm{CH}_{2} \mathrm{Cl}_{2}\right)$ as eluent to remove impurities and then dried over $3 \AA$ molecular sieves in $\mathrm{CHCl}_{3}$. The lactide monomers were further purified by recrystallization in toluene before being stored into a glovebox with inert atmosphere. (-)-Sparteine was dried over $\mathrm{CaH}_{2}$ before use and 1-(3,5-bis(trifluoromethyl) phenyl)-3-cyclohexyl-thiourea was prepared and purified as reported ${ }^{41}$. THPA was synthesized and purified as described previously ${ }^{42}$. 2,2-azobis(isobutyronitrile) was recrystallized from methanol and stored at $4{ }^{\circ} \mathrm{C}$. PEG was purchased from Iris Biotech and dried in a desiccator under vacuum over $\mathrm{P}_{2} \mathrm{O}_{5}$.

Instrumentation. ${ }^{1} \mathrm{H}$ NMR spectra were recorded on Bruker spectrometers operating at a frequency of 300 or $400 \mathrm{MHz}$ in $\mathrm{CDCl}_{3}$. The chemical shifts are given in ppm with tetramethylsilane as internal reference. SEC was performed on an Agilent 1,260 Infinity Multi-Detector SEC instrument equipped with refractive index and ultraviolet detectors with THF and 2\% triethyl amine as eluent at a flow rate of $1 \mathrm{ml} \mathrm{min}^{-1}$. SEC data were calibrated by Cirrus GPC software with poly(styrene) standards.

FT-IR spectra were obtained using a Perkin Elmer Spectrum 100 FT-IR. Scans from 550 to $4,000 \mathrm{~cm}^{-1}$ were taken, and the spectra corrected for background absorbance, all curves were normalized.

Mass spectra were obtained by using a Bruker Ultraflex II Matrix-assisted laser desorption/ionization time of flight (MALDI-ToF) mass spectrometer. The MALDI-ToF samples were prepared as follows: trans-2-[3-(4-tert-butyl-phenyl)-2methyl-2-propenylidene] malononitrile (DCTB) was used as a matrix while sodium trifluoroacetate was used as a cationization agent. Typically, DCTB $(20 \mu \mathrm{l}$ of a $40 \mathrm{mg} \mathrm{ml}^{-1}$ HPLC grade THF solution), samples ( $20 \mu \mathrm{l}$ of a $1 \mathrm{mg} \mathrm{ml}^{-1}$ HPLC grade THF solution) and sodium trifluoroacetate ( $20 \mu \mathrm{l}$ of a $0.1 \mathrm{mg} \mathrm{ml}^{-1}$ HPLC grade THF solution) were successively added into a small centrifuge tube and mixed by a vortex mixer. Traces of mixture were deposited on a MALDI-ToF plate followed by solvent evaporation. The samples were measured in reflectron ion mode and calibrated by SpheriCal $\left(1,200 \sim 8,000 \mathrm{~g} \mathrm{~mol}^{-1}\right)$ standards.

The hydrodynamic diameter $\left(D_{\mathrm{h}}\right)$ of different nanoparticles was determined by DLS. Typically, $0.25 \mathrm{mg} \mathrm{ml}^{-1}$ aqueous nanoparticle solution scattering was measured in a Malvern Zetasizer NanoS instrument equipped with a $4 \mathrm{~mW} \mathrm{He}-\mathrm{Ne}$ 633 -nm laser module at $25^{\circ} \mathrm{C}$. Measurements were carried out at a detection angle of $173^{\circ}$ (back scattering) and the data were further analyzed by Malvern DTS 6.20 software. $D_{\mathrm{h}}$ was calculated by fitting the apparent diffusion coefficient in the Stokes-Einstein equation $D_{\mathrm{h}}=k T /\left(3 \pi \eta D_{\text {app }}\right)$, where $k$ is the Boltzmann constant, $T$ is the temperature and $\eta$ is the viscosity of the solvent. $D_{\mathrm{h}}$ only coincides to the real hydrodynamic diameter when the measured sample is a solution of monodispersed spherical particles as $D_{\text {app }}$ equals the translational diffusion $\left(D_{\mathrm{t}}\right)$. For cylindrical particles, owing to their anisotropy, the rotational diffusion is not negligible and contributes to the $D_{\mathrm{app}}$. Therefore, the $D_{\mathrm{h}}$ measured for the cylindrical micelles only has a relative value and provides polydispersity information to detect multiple populations.

TEM images on GO support (Fig. 5) were obtained by using an aberrationcorrected JEOL JEM-ARM200F instrument operating at $80 \mathrm{kV}$ with spherical aberration corrected tuned to $\sim+1 \mu \mathrm{m}$ and images were recorded on a Gatan SC-1,000 Orius CCD camera. TEM samples were prepared using a freeze-drying method using GO-covered TEM grids that provide a thin support, which is almost electron transparent and gives excellent contrast ${ }^{28}$. Generally, one drop of each sample solution $\left(5 \mu \mathrm{l}\right.$ of $0.1 \mathrm{mg} \mathrm{ml}^{-1}$ ) was added onto the $\mathrm{GO}$ grid and was frozen using liquid nitrogen for $15 \mathrm{~s}$. The grid was then placed into a small vial inside a flask, which could be attached to a freeze drier. After lyophilization, the grid was collected for TEM analysis. Analysis was performed on a minimum of 20 particles of each type with 20 images in each focal series. The stained TEM images were obtained by using a JEOL $2000 \mathrm{FX}$ instrument operated at $200 \mathrm{kV}$. TEM samples were negatively stained by (phosphotungstic acid, $2 \mathrm{wt} \%$ ) on formvar/carbon grid (300 Mesh, Cu, Elektron Technology UK LTD). Typically, formvar/carbon grids were cleaned by air plasma from a glow-discharge system $(2 \mathrm{~min}, 20 \mathrm{~mA})$, which also improved the hydrophilicity character of the grids. $20 \mu \mathrm{l}$ of particle solution $\left(0.25 \mathrm{mg} \mathrm{ml}^{-1}\right)$ was added onto the grid and the solution was blotted away after $2 \mathrm{~min}$ and then left to air dry. Five microlitres of a $2 \mathrm{wt} \%$ PTA solution was then added on the grid to stain the particles and was blotted away after $30 \mathrm{~s}$ before air drying. TEM images were analyzed by ImageJ software, and 200 particles were counted for each sample to obtain the number-average length $\left(L_{\mathrm{n}}\right)$ and to calculate weight-average length $\left(L_{\mathrm{w}}\right)$, number-average width $\left(W_{\mathrm{n}}\right)$ (for cylindrical micelles) and number-average diameter $\left(D_{\mathrm{n}}\right)$ (for spherical micelles). $L_{\mathrm{n}}, L_{\mathrm{w}}, W_{\mathrm{n}}$ and $D_{\mathrm{n}}$ were calculated by using the following equations:

$$
\begin{gathered}
L_{\mathrm{n}}=\frac{\sum_{\mathrm{i}=1}^{\mathrm{n}} N_{\mathrm{i}} L_{\mathrm{i}}}{\sum_{\mathrm{i}=1}^{\mathrm{n}} N_{\mathrm{i}}} \\
L_{\mathrm{w}}=\frac{\sum_{\mathrm{i}=1}^{\mathrm{n}} N_{\mathrm{i}} L_{\mathrm{i}}^{2}}{\sum_{\mathrm{i}=1}^{\mathrm{n}} N_{\mathrm{i}} L_{\mathrm{i}}} \\
W_{\mathrm{n}}=\frac{\sum_{\mathrm{i}=1}^{\mathrm{n}} N_{\mathrm{i}} W_{\mathrm{i}}}{\sum_{\mathrm{i}=1}^{\mathrm{n}} N_{\mathrm{i}}} \\
D_{\mathrm{n}}=\frac{\sum_{\mathrm{i}=1}^{\mathrm{n}} N_{\mathrm{i}} D_{\mathrm{i}}}{\sum_{\mathrm{i}=1}^{\mathrm{n}} N_{\mathrm{i}}}
\end{gathered}
$$

where $L_{\mathrm{i}}$ and $W_{\mathrm{i}}$ are the length and the width of each counted cylindrical micelle, whereas $D_{\mathrm{i}}$ is the diameter of each counted spherical micelle. $N_{\mathrm{i}}$ is the number of the cylindrical micelles with the length of $L_{\mathrm{i}}$ and the width of $W_{\mathrm{i}}$ or the number of the spherical micelles with the diameter of $D_{\mathrm{i}}$.

SAXS were carried on the SAXS/WAXS beamline at the Australian Synchrotron facility at a photon energy of $11 \mathrm{keV}$. The samples in solution were run using $1.5 \mathrm{~mm}$ diameter quartz capillaries. The reactions took place in heated vials, with the solutions circulating through the capillaries using a peristaltic pump. Such a setup allows living data to be collected in situ, which provides a real-time insight into the overall self-assembly process and the evolution of the morphologies. The measurements were collected at a sample to detector distance of $7.364 \mathrm{~m}$ to give a $q$ range of $0.002-0.08 \AA^{-1}$ (SAXS), where $q$ is the scattering vector and is related to the scattering angle $(2 \theta)$ and the photon wavelength $(\lambda)$ by the following equation:

$$
q=\frac{4 \pi \sin \theta}{\lambda}
$$

Data were processed using ScatterBrain for radial integration, normalization, absolute scaling and background subtraction: patterns were normalized to fixed transmitted flux using a quantitative beamstop detector; the scattering from a blank $\left(\mathrm{H}_{2} \mathrm{O}\right)$ was measured in the same capillary and was subtracted for each measurement; the two-dimensional SAXS images were converted in onedimensional SAXS profile $(I(q)$ versus $q$ ) by circular averaging, where $I(q)$ is the scattering intensity. NIST SANS macros were used for SAXS data analysis using Igor Pro software ${ }^{43}$. The scattering length density of the solvent was calculated using the scattering length density calculator provided by the NIST Center for Neutron Research ${ }^{44}$. Limits for $q$ range were applied for the fitting of SAXS data from 0.002 to $0.07 \AA$.

WAXD was performed on a Panalytical X'Pert Pro MPD equipped with a $\mathrm{CuK} \alpha_{1}$ hybrid monochromator as the incident beam optics. Generally, $\sim 30 \mathrm{mg}$ of self-assembled freeze-dried particles were placed in a $10 \mathrm{~mm}$ sample holder, and standard powder $2 \theta-\theta$ diffraction scans were carried out in the angular range from $2 \theta 10^{\circ}$ to $30^{\circ}$ at room temperature.

Synthesis of dodecyl 4-(hydroxymethyl) benzyl carbonotrithioate. The dualheaded initiator was synthesized as reported previously ${ }^{22}$. Generally, dodecanethiol $(1.29 \mathrm{~g}, 6.4 \mathrm{mmol})$, potassium phosphate $(1.48 \mathrm{~g}, 7 \mathrm{mmol})$ and carbon disulphide $(1.14 \mathrm{ml}, 19 \mathrm{mmol})$ were added into acetone $(200 \mathrm{ml})$. After stirring for $2 \mathrm{~h}$ at room temperature, 4-chloromethylbenzyl alcohol $(1.00 \mathrm{~g}, 6.4 \mathrm{mmol})$ was added into the solution. The mixture was allowed to stir for another $24 \mathrm{~h}$. Acetone was then removed in vacuo and the resultant solid was dissolved in $\mathrm{CHCl}_{3}$. The organic layer was washed against $\mathrm{HCl}\left(1 \mathrm{moll}^{-1}, 200 \mathrm{ml} \times 1\right)$, distilled water $(200 \mathrm{ml} \times 3)$ and brine $(200 \mathrm{ml} \times 1)$ before being dried over magnesium sulfate. Solids were removed 
by filtration and the solution was concentrated in vacuo before purification through a silica column $(n$-hexane/ethyl acetate $=60 / 40)$. The solvent was removed in vacuo to yield a yellow solid, which was dried over $\mathrm{P}_{2} \mathrm{O}_{5}$ in a dessicator for 2 days before being transferred into a glove box with inert atmosphere. ${ }^{1} \mathrm{H}$ NMR $\left(400 \mathrm{MHz}, \mathrm{CDCl}_{3}\right) \delta 7.40-7.28(4 \mathrm{H}, \mathrm{m}), 4.68\left(2 \mathrm{H}, \mathrm{d},{ }^{3} \mathrm{~J}_{\mathrm{H}-\mathrm{H}}=5.0 \mathrm{~Hz}\right), 4.61(2 \mathrm{H}, \mathrm{s})$, $3.37\left(2 \mathrm{H}, \mathrm{t},{ }^{3} \mathrm{~J}_{\mathrm{H}-\mathrm{H}}=7.5 \mathrm{~Hz}\right), 1.77-1.58(3 \mathrm{H}, \mathrm{m}), 1.46-1.20(18 \mathrm{H}, \mathrm{m}), 0.88(3 \mathrm{H}, \mathrm{t}$, $\left.{ }^{3} J_{\mathrm{H}-\mathrm{H}}=7.0 \mathrm{~Hz}\right) ;{ }^{3} \mathrm{C}$ NMR $\left(150 \mathrm{MHz}, \mathrm{CDCl}_{3}\right) \delta 223.7,140.4,134.6,129.5,127.3$, $65.0,41.0,37.1,32-22,14.1$.

ROP of $L$-lactide ( $D$-lactide). PLLA (or PDLA) was synthesized in a glove box under nitrogen atmosphere as reported previously $y^{22,25}$. Typically, for PLLA $(\mathrm{DP}=31)$, the dual-headed initiator $(92.7 \mathrm{mg}, 0.23 \mathrm{mmol})$ and $(-)$-sparteine $(40.1 \mu \mathrm{l}, 0.17 \mathrm{mmol})$ were combined in one vial with L-lactide $(1.01 \mathrm{~g}, 6.98 \mathrm{mmol})$ and 1-(3,5-bis(trifluoromethyl)phenyl)-3-cyclohexyl-thiourea (129.2 mg, $0.35 \mathrm{mmol}$ ) in another. $\mathrm{CHCl}_{3}$ (4 and $6 \mathrm{ml}$, respectively) was then added to each of the vials before the two solutions were mixed and allowed to stir at room temperature for $3 \mathrm{~h}$. The product was precipitated in $n$-hexane three times before filtration and drying in vacuo to yield a pale yellow solid. ${ }^{1} \mathrm{H}$ NMR $(400 \mathrm{MHz}$, $\left.\mathrm{CDCl}_{3}\right) \delta$ 7.36-7.30 $(4 \mathrm{H}, \mathrm{m}), 5.40-5.30(2 \mathrm{H}$ PLLA $+2 \mathrm{H}, \mathrm{m}), 4.61(2 \mathrm{H}, \mathrm{s}), 3.37$ $\left(2 \mathrm{H}, \mathrm{t},{ }^{3} \mathrm{~J}_{\mathrm{H}-\mathrm{H}}=7.5 \mathrm{~Hz}\right), 1.66-1.48(6 \mathrm{H}$ PLLA $+2 \mathrm{H}, \mathrm{m}), 1.34-1.18(18 \mathrm{H}, \mathrm{br}), 0.88$ $\left(3 \mathrm{H}, \mathrm{t},{ }^{3} \mathrm{~J}_{\mathrm{H}-\mathrm{H}}=6.5 \mathrm{~Hz}\right)$.

Synthesis of PLLA-b-PTHPA 1 (PDLA-b-PTHPA 2). The representative procedure of the synthesis of diblock copolymer 1 is as follows ${ }^{22,25}$. Typically, PLLA ${ }_{31}$ macro-initiator (194.7 mg, 1 eq.) and THPA (2.5 g, 400 eq.) monomer were dissolved in $\mathrm{CHCl}_{3}(2.5 \mathrm{ml})$ and transferred into a dried ampoule before addition of azobis(isobutyronitrile) $\left(65.8 \mu \mathrm{l}\right.$ of a $10 \mathrm{mg} \mathrm{ml}^{-1} \mathrm{CHCl}_{3}$ solution). The solution was degassed by three freeze-pump-thaw cycles and sealed under argon and then placed in a $60^{\circ} \mathrm{C}$ oil bath with stirring for $3.5 \mathrm{~h}$. The product was precipitated into $n$-hexane for three times and dried in vacuo to give a pale yellow solid $(76 \%$ conversion by ${ }^{1} \mathrm{H}$ NMR spectroscopy) with a final yield of $98 \% .{ }^{1} \mathrm{H}$ NMR $\left(400 \mathrm{MHz}, \mathrm{CDCl}_{3}\right) \delta 6.20-5.68$ (1H PTHPA, br), 5.26-5.04 (2H PLLA, m), 3.96-3.58 (2H PTHPA, br), 2.66-2.24 ( ${ }^{1}$ H PTHPA, br), 2.18-1.36 (8H PTHPA \& $6 \mathrm{H}$ PLLA, br m).

ROP of $L$-lactide ( $D$-lactide) from PEG(20k). PEG-PLLA (or PEG-PDLA) was synthesized in a glove box under nitrogen atmosphere as reported previously ${ }^{31}$. Typically, for PLLA ( $D P=27)$, the PEG monomethyl ether initiator $(300 \mathrm{mg}$, $0.015 \mathrm{mmol})$ and $(-)$-sparteine $(2.67 \mu \mathrm{l}, 0.012 \mathrm{mmol})$ were combined in one vial with $L$-lactide $(67 \mathrm{mg}, 0.465 \mathrm{mmol})$ and 1-(3,5-bis(trifluoromethyl)phenyl)-3cyclohexyl-thiourea $(8.6 \mathrm{mg}, 0.023 \mathrm{mmol})$ in another. $\mathrm{CH}_{2} \mathrm{Cl}_{2}(1.6$ and $0.3 \mathrm{ml}$, respectively) was then added to each of the vials before the two solutions were mixed and allowed to stir at room temperature for $31 \mathrm{~h}$. The product was precipitated in cold diethyl ether three times before filtration and drying in vacuo to yield a pale white solid. ${ }^{1} \mathrm{H}$ NMR $(300 \mathrm{MHz}, \mathrm{CDCl} 3) \delta 5.21-5.13$ (2H PLLA, q, ${ }^{3} \mathrm{~J}_{\mathrm{H}-\mathrm{H}}=7.4 \mathrm{~Hz}$ ), 3.64 (4H PEG, s), $3.38\left(3 \mathrm{H} \mathrm{CH} \mathrm{CH}_{3} \mathrm{O}-\mathrm{PEG}, \mathrm{s}\right), 1.60-1.66$ (6H PLLA, d, ${ }^{3} J_{\mathrm{H}-\mathrm{H}}=6.2 \mathrm{~Hz}$ )

Preparation of amorphous spheres from diblock copolymer 1. Diblock copolymer $1(50 \mathrm{mg})$ was exposed to the identical CDSA conditions $\left(v_{\mathrm{THF}} / v_{\mathrm{H} 2 \mathrm{O}}=20 / 80\right.$, acetic acid (1 eq. per THPA block), evaporation of THF, $\left.65^{\circ} \mathrm{C}\right)^{22,25}$. After $2.5 \mathrm{~h}$ of self-assembly, as the morphology of the nanostructures was still a spherical structure (before the morphological transition into cylinder) ${ }^{24}$, the nanoparticle solution was quenched by cooling in liquid nitrogen and subsequently lyophilized. The spherical morphology was confirmed by using high resolution transmission electron microscope (Fig. 5e), and the amorphous core nature of these nanoparticles was evidenced by using WAXD (Supplementary Fig. S14).

Self-assembly of $\mathbf{1}$ and $\mathbf{2}$ to realize stereocomplex particles. Identical selfassembly conditions that have been previously reported by the group to afford homochiral cylinders were utilized to obtain stereocomplex particles ${ }^{22,25}$. A 1:1 mixture of $1(25 \mathrm{mg})$ and $2(25 \mathrm{mg})$ were added in $0.5 \mathrm{ml}$ THF to $2 \mathrm{ml}$ of $\mathrm{H}_{2} \mathrm{O}$ (resistivity $18.2 \mathrm{M} \Omega \cdot \mathrm{cm})(v / v=20 / 80)$ in a vial and sealed with a needle through the seal. Acetic acid (1 eq. per THPA block) was added into the mixture and the self-assembly was set up at $65^{\circ} \mathrm{C}$. After $30 \mathrm{~h}$, the solution was quenched by cooling in liquid nitrogen and subsequently lyophilized. The freeze-dried particles were then redispersed in $\mathrm{H}_{2} \mathrm{O}\left(0.25 \mathrm{mg} \mathrm{ml}^{-1}\right)$ at room temperature for TEM and DLS analysis.

CDSA of 1 or 2. The identical CDSA conditions described above were used to prepare cylindrical micelle 3 or 4 from either diblock copolymer 1 or 2 as previously reported ${ }^{22,25}$. Generally, $0.5 \mathrm{ml}$ THF and $2 \mathrm{ml} \mathrm{H}_{2} \mathrm{O}$ (resistivity $18.2 \mathrm{M} \Omega \cdot \mathrm{cm}$ ) were added into $50 \mathrm{mg}$ polymer inside a vial. Acetic acid (1 eq. per THPA block) was also added to the mixture. The vial was sealed with a needle through the seal and the mixture was allowed to stir at $65^{\circ} \mathrm{C}$ for $30 \mathrm{~h}$ before being quenched in liquid nitrogen and subsequent lyophilization. The freeze-dried particles were then dissolved directly into $\mathrm{H}_{2} \mathrm{O}\left(0.25 \mathrm{mg} \mathrm{ml}^{-1}\right)$ at room temperature for TEM and DLS analysis.

Addition of PDLA-b-PAA unimers into PLLA-b-PAA cylinder 3. Diblock copolymer $2(50 \mathrm{mg})$ was exposed to the identical CDSA conditions $\left(v_{\mathrm{THF}} /\right.$ $v_{\mathrm{H} 2 \mathrm{O}}=20 / 80$, acetic acid (1 eq. per THPA block), evaporation of THF, $65^{\circ} \mathrm{C}$ ). After $2.5 \mathrm{~h}$, as the PTHPA block was completely hydrolyzed into PAA block ${ }^{24}$, the solution was quenched by cooling in liquid nitrogen and subsequently lyophilized. The freeze-dried PDLA- $b$-PAA diblock copolymer $(17 \mathrm{mg})$ was fully dissolved in THF $(0.5 \mathrm{ml})$. DLS analysis confirmed that PDLA- $b$-PAA existed as unimer state in THF (Supplementary Fig. S22g). THF solution $(0.5 \mathrm{ml})$ of PDLA- $b$-PAA unimers was then added into $2 \mathrm{ml}$ aqueous solution of PLLA- $b$-PAA cylinder $3(17 \mathrm{mg})$ in a vial. The vial was sealed with a needle through the seal and the mixture was allowed to stir at $65^{\circ} \mathrm{C}$. At different time intervals, the mixture was sampled and quenched by cooling in liquid nitrogen and subsequently lyophilized. The freeze-dried particles were then dissolved directly into $\mathrm{H}_{2} \mathrm{O}\left(0.25 \mathrm{mg} \mathrm{ml}^{-1}\right)$ at room temperature for TEM and DLS analysis.

Mixture of PLLA-b-PEG cylinder and PDLA-b-PEG cylinder. The homochiral PLLA- $b$-PEG cylinder and PDLA- $b$-PEG cylinder were prepared as previously reported by the group ${ }^{31}$. One millilitre of each cylinder solution (note: the cylinder solution still co-existed with a number of spherical micelles) was directly mixed $\left(20 \mathrm{mg} \mathrm{ml}^{-1}\right)$ before addition of $0.5 \mathrm{ml}$ THF. The mixture was sealed with a needle through the seal and the mixture was allowed to stir at $65^{\circ} \mathrm{C}$. At different time intervals, the mixture was sampled and quenched by cooling in liquid nitrogen and subsequently lyophilized. The freeze-dried particles were analyzed by FT-IR and dissolved directly into $\mathrm{H}_{2} \mathrm{O}\left(0.25 \mathrm{mg} \mathrm{ml}^{-1}\right)$ at room temperature for TEM analysis.

\section{References}

1. Jeong, B., Bae, Y. H., Lee, D. S. \& Kim, S. W. Biodegradable block copolymers as injectable drug-delivery systems. Nature 388, 860-862 (1997).

2. Tsuji, H. Poly(lactide) stereocomplexes: formation, structure, properties, degradation, and applications. Macromol. Biosci. 5, 569-597 (2005).

3. Oh, J. K. Polylactide (PLA)-based amphiphilic block copolymers: synthesis, self-assembly, and biomedical applications. Soft Matter 7, 5096-5108 (2011).

4. Albertsson, A.-C. \& Varma, I. K. Recent developments in ring opening polymerization of lactones for biomedical applications. Biomacromolecules 4, 1466-1486 (2003).

5. Uhrich, K. E., Cannizzaro, S. M., Langer, R. S. \& Shakesheff, K. M. Polymeric systems for controlled drug release. Chem. Rev. 99, 3181-3198 (1999).

6. Ikada, Y., Jamshidi, K., Tsuji, H. \& Hyon, S. H. Stereocomplex formation between enantiomeric poly(Lactides). Macromolecules 20, 904-906 (1987).

7. Zhang, J., Sato, H., Tsuji, H., Noda, I. \& Ozaki, Y. Infrared spectroscopic study of $\mathrm{CH} 3 \cdots \mathrm{O}=\mathrm{C}$ interaction during poly $(L$-lactide $) / \operatorname{poly}(D$-lactide $)$ stereocomplex formation. Macromolecules 38, 1822-1828 (2005).

8. Tsuji, H. \& Ikada, Y. Stereocomplex formation between enantiomeric poly(lactic acid)s. XI. Mechanical properties and morphology of solution-cast films. Polymer 40, 6699-6708 (1999).

9. Fukushima, K. \& Kimura, Y. Stereocomplexed polylactides (Neo-PLA) as highperformance bio-based polymers: their formation, properties, and application. Polym. Int. 55, 626-642 (2006).

10. Portinha, D. et al. Stable dispersions of highly anisotropic nanoparticles formed by cocrystallization of enantiomeric diblock copolymers. Macromolecules $\mathbf{4 0}$, 4037-4042 (2007).

11. Nagahama, K., Mori, Y., Ohya, Y. \& Ouchi, T. Biodegradable nanogel formation of polylactide-grafted dextran copolymer in dilute aqueous solution and enhancement of its stability by stereocomplexation. Biomacromolecules $\mathbf{8}$, 2135-2141 (2007).

12. Kang, N. et al. Stereocomplex block copolymer micelles: core-shell nanostructures with enhanced stability. Nano Lett. 5, 315-319 (2005).

13. Kim, S. H. et al. Mixed micelle formation through stereocomplexation between enantiomeric poly(lactide) block copolymers. Macromolecules 42, 25-29 (2009).

14. Nederberg, F. et al. Simple approach to stabilized micelles employing miktoarm terpolymers and stereocomplexes with application in paclitaxel delivery. Biomacromolecules 10, 1460-1468 (2009).

15. Tan, B. H. et al. Tuning self-assembly of hybrid PLA-P(MA-POSS) block copolymers in solution via stereocomplexation. Polym. Chem. 4, 1250-1259 (2013).

16. Wolf, F. K., Hofmann, A. M. \& Frey, H. Poly(isoglycerol methacrylate)- $b$ poly(D or L-lactide) copolymers: a novel hydrophilic methacrylate as building block for supramolecular aggregates. Macromolecules 43, 3314-3324 (2010).

17. Portinha, D., Bouteiller, L., Pensec, S., Richez, A. \& Chassenieux, C. Influence of preparation conditions on the self-assembly by stereocomplexation of polylactide containing diblock copolymers. Macromolecules 37, 3401-3406 (2004). 
18. Han, Y. \& Jiang, W. Self-assembly of the AB/BC diblock copolymer mixture based on hydrogen bonding in a selective solvent: a Monte Carlo study. J. Phys. Chem. B 115, 2167-2172 (2011).

19. Palyulin, V. V. \& Potemkin, I. I. Mixed versus ordinary micelles in the dilute solution of AB and BC diblock copolymers. Macromolecules 41, 4459-4463 (2008).

20. Hu, J. W. \& Liu, G. J. Chain mixing and segregation in B-C and C-D diblock copolymer micelles. Macromolecules 38, 8058-8065 (2005).

21. Liu, T., Qian, Y., Hu, X., Ge, Z. \& Liu, S. Mixed polymeric micelles as multifunctional scaffold for combined magnetic resonance imaging contrast enhancement and targeted chemotherapeutic drug delivery. J. Mater. Chem. 22, 5020-5030 (2012).

22. Petzetakis, N., Dove, A. P. \& O’Reilly, R. K. Cylindrical micelles from the living crystallization-driven self-assembly of poly(lactide)-containing block copolymers. Chem. Sci. 2, 955-960 (2011).

23. Petzetakis, N. et al. Hollow block copolymer nanoparticles through a spontaneous one-step structural reorganization. ACS Nano 7, 1120-1128 (2013).

24. Petzetakis, N., Walker, D., Dove, A. P. \& O’Reilly, R. K. Crystallization-driven sphere-to-rod transition of poly(lactide)- $b$-poly(acrylic acid) diblock copolymers: mechanism and kinetics. Soft Matter 8, 3408-3414 (2012).

25. Sun, L. et al. Tuning the size of cylindrical micelles from poly( $L$-lactide)- $b$ poly(acrylic acid) diblock copolymers based on crystallization-driven self-assembly. Macromolecules 46, 9074-9082 (2013).

26. Schmelz, J., Karg, M., Hellweg, T. \& Schmalz, H. General pathway toward crystalline-core micelles with tunable morphology and corona segregation. ACS Nano 5, 9523-9534 (2011).

27. Becker, J. M., Pounder, R. J. \& Dove, A. P. Synthesis of poly(lactide)s with modified thermal and mechanical properties. Macromol. Rapid Commun. 31, 1923-1937 (2010).

28. Patterson, J. P. et al. A simple approach to characterizing block copolymer assemblies: graphene oxide supports for high contrast multi-technique imaging. Soft Matter 8, 3322-3328 (2012).

29. Dyson, M. A. et al. A new approach to high resolution, high contrast electron microscopy of macromolecular block copolymer assemblies. Soft Matter 9, 3741-3749 (2013).

30. Hsiao, M.-S., Yusoff, S. F. M., Winnik, M. A. \& Manners, I. Crystallization-driven self-assembly of block copolymers with a short crystallizable core-forming segment: controlling micelle morphology through the influence of molar mass and solvent selectivity. Macromolecules 47, 2361-2372 (2014)

31. Pitto-Barry, A., Kirby, N., Dove, A. P. \& O’Reilly, R. K. Expanding the scope of the crystallization-driven self-assembly of polylactide-containing polymers. Polym. Chem. 5, 1427-1436 (2014).

32. Zhang, L. F. \& Eisenberg, A. Thermodynamic vs kinetic aspects in the formation and morphological transitions of crew-cut aggregates produced by self-assembly of polystyrene- $b$-poly(acrylic acid) block copolymers in dilute solution. Macromolecules 32, 2239-2249 (1999).

33. Zhang, J., Wang, L. Q., Wang, H. J. \& Tu, K. H. Micellization phenomena of amphiphilic block copolymers based on methoxy poly(ethylene glycol) and either crystalline or amorphous poly(caprolactone- $b$-lactide). Biomacromolecules 7, 2492-2500 (2006).

34. Blanazs, A., Madsen, J., Battaglia, G., Ryan, A. J. \& Armes, S. P. Mechanistic insights for block copolymer morphologies: how do worms form vesicles? J. Am. Chem. Soc. 133, 16581-16587 (2011).

35. Wang, X. S. et al. Cylindrical block copolymer micelles and co-micelles of controlled length and architecture. Science 317, 644-647 (2007).

36. Gilroy, J. B. et al. Monodisperse cylindrical micelles by crystallization-driven living self-assembly. Nat. Chem. 2, 566-570 (2010).

37. Schmelz, J., Schedl, A. E., Steinlein, C., Manners, I. \& Schmalz, H. Length control and block-type architectures in worm-like micelles with polyethylene cores. J. Am. Chem. Soc. 134, 14217-14225 (2012).
38. Qian, J. et al. Uniform, high aspect ratio fiber-like micelles and block comicelles with a crystalline pi-conjugated polythiophene core by self-seeding. J. Am. Chem. Soc. 136, 4121-4124 (2014).

39. Qian, J. et al. Self-seeding in one dimension: a route to uniform fiber-like nanostructures from block copolymers with a crystallizable core-forming block. ACS Nano 7, 3754-3766 (2013).

40. Brizzolara, D., Cantow, H.-J., Diederichs, K., Keller, E. \& Domb, A. J. Mechanism of the stereocomplex formation between enantiomeric poly(lactide)s. Macromolecules 29, 191-197 (1996).

41. Pratt, R. C. et al. Exploration, optimization, and application of supramolecular thiourea-amine catalysts for the synthesis of lactide (co)polymers. Macromolecules 39, 7863-7871 (2006).

42. Hertler, W. R. Catalyzed process for reacting carboxylic acids with vinyl ethers. US Patent 5072029 (1991).

43. Kline, S. Reduction and analysis of SANS and USANS data using IGOR Pro. J. Appl. Crystalogr. 39, 895-900 (2006).

44. Scattering Length Density Calculator http://www.ncnr.nist.gov/resources/ sldcalc.html (2014)

\section{Acknowledgements}

We thank the University of Warwick, Swiss National Science Foundation and the EPSRC for funding. The Royal Society is thanked for an Industry Fellowship to A.P.D. The Engineering and Physical Sciences Research Council (EP/G004897/1) are thanked for funding to support postdoctoral fellowships for A.P.B. as well as funding for J.S. and M.A.D. through the Warwick Centre for Analytical Science (EP/F034210/1). A.M.S. thanks the Science City Research Alliance and the HEFCE Strategic Development Fund for funding support. Some items of equipment that were used in this research were funded by Birmingham Science City, with support from Advantage West Midlands and part-funded by the European Regional Development Fund.

\section{Author contributions}

L.S. designed the experiments, performed the experiments, analyzed and discussed the results. A.P.B. performed the SAXS analysis, some synthesis and characterization, discussed the results and commented on the paper. N.K. and T.S. designed the SAXS experiments and in situ analysis rig, assisted with SAXS analysis and commented on the paper. M.A.D. and A.S. performed the high-resolution microscopy and its EWR analysis and discussed the results and commented on the paper. N.R.W. and J.S. discussed the microscopy results and commented on the paper. A.P.D. and R.K.O.R. conceived and obtained funding for the project, designed experiments and discussed the results. The paper was written by L.S, A.P.D. and R.K.O.R.

\section{Additional information}

Supplementary Information accompanies this paper at http://www.nature.com/ naturecommunications

Competing financial interests: The authors declare no competing financial interest.

Reprints and permission information is available online at http://npg.nature.com/ reprintsandpermissions/

How to cite this article: Sun, L. et al. Structural reorganization of cylindrical nanoparticles triggered by polylactide stereocomplexation. Nat. Commun. 5:5746 doi: $10.1038 /$ ncomms6746 (2014).

This work is licensed under a Creative Commons Attribution 4.0 International License. The images or other third party material in this article are included in the article's Creative Commons license, unless indicated otherwise in the credit line; if the material is not included under the Creative Commons license, users will need to obtain permission from the license holder to reproduce the material. To view a copy of this license, visit http://creativecommons.org/licenses/by/4.0/ 